\title{
DESARROLLO INFANTIL Y NUTRICIÓN. ¿QUÉ SABEN LOS ESTUDIANTES UNIVERSITARIOS?
}

\author{
CHILD DEVELOPMENT AND \\ NUTRITION. WHAT DO \\ UNIVERSITY STUDENTS KNOW?
}

Recibido 4 de junio 2017 Aceptado 13 de octubre 2017

Correspondencia: María Magdalena Álvarez Ramírez Médicos y Odontólogos S/N Unidad del Bosque. C. P. 91017 Xalapa, Veracruz, México. Tel. 012288421700 Ext. 16555 E-mail: malvarez@uv.mx

Palabras clave: conocimiento, desarrollo infantil, nutrición, estudiantes universitarios

Key words: knowledge, child development, nutrition, university students.

\section{Autores:}

Álvarez Ramírez María Magdalena

Facultad de Nutrición-Xalapa, Universidad Veracruzana Laboratorio de Psicobiología

Granados Ramos Dora Elizabeth

Facultad Psicología-Xalapa, Universidad Veracruzana, Laboratorio de Psicobiología. 


\section{RESUMEN}

Introducción: Los estudiantes universitarios que se desempeñarán en la atención del binomio madreniño deben reconocer la importancia que tiene el conocimiento sobre Desarrollo infantil, Nutrición y Alimentación en la atención temprana.

Objetivo: Analizar qué saben sobre desarrollo infantil, nutrición y alimentación los estudiantes universitarios

Material y Métodos: Estudio exploratorio mediante la realización de una encuesta semiestructurada en una población de 134 estudiantes de las licenciaturas de: Enfermería, Nutrición, Medicina, Psicología y Pedagogía de la Universidad Veracruzana. Los datos se procesaron en el programa Minitab 15 aplicándose pruebas de Kruskal Wallis

Resultados: Los estudiantes mostraron dificultad a expresar sus conocimientos y el nivel de estos no fue homogéneo en los grupos, Las puntuaciones sobre desarrollo infantil fueron más altas en los estudiantes de Enfermería y Medicina, sin embargo, en todos los estudiantes, el conocimiento acerca de los hitos del desarrollo y Nutrición fue inferior a lo recomendado con una mediana de 5 de 10 y 3 de 18 aciertos respectivamente.

Discusión y Conclusiones: El estudio reportó conocimientos insuficientes por lo que se debe promover que los estudiantes de licenciatura se apropien del conocimiento, favoreciendo que evalúen aspectos del desarrollo Infantil y Nutrición de manera cotidiana considerando la interacción con los padres de familia, a fin de que experimenten afinidad por el tema, profundicenen los conceptosy, de esta manera, mejoren su desempeño profesional permitiéndole identificar a los niños que presentan trastornos en su desarrollo o tienen riesgo de padecerlos. Se sugiere la creación de una unidad multidisciplinaria de Nutrición y Desarrollo infantil como espacio inter y transdisciplinar donde se brinden estrategias educativas a los futuros profesionistas involucrados en la atención temprana.

Palabras claves: conocimiento, desarrollo infantil, nutrición, estudiantes universitarios.

72 MMN $\mathrm{WH}_{W}$ Enf Neurol Vol. 16. No. 2 mayo - agosto 2017
ABSTRACT

Introduction: University students who will serve in the care of the mother-child binomial should recognize the importance of knowledge about Child Development, Nutrition and Food in early care.

Objective: To analyze what university students know about child development, nutrition and feeding.

Material and Methods: Exploratory study by a semi structured survey in a population of 134 undergraduate students of: Nursing, Nutrition, Medicine, Psychology and Pedagogy of the Veracruz University. The data were processed in the program Minitab 15 applying tests of Kruskal Wallis.

Results: Students showed difficulty in expressing their knowledge and their level was not homogeneous in the groups. Child development scores were higher in Nursing and Medicinestudents, however in all students, knowledge about the Development milestones and Nutrition was lower than recommended with a median of 5 of 10 and 3 of 18 successes respectively.

Discussion and conclusions: The study reported insufficient knowledge and it should be encouraged that undergraduate students take ownership of knowledge, favoring that they evaluate aspects of child development and nutrition in a daily way considering the interaction with parents, so that experience affinity for the subject, deepen the concepts and thus improve their professional performance allowing them to identify children who have developmental disorders or are at risk of suffering them. It is suggested the creation of a multidisciplinary unit of Nutrition and Child Development as an inter and transdisciplinary space where educational strategies are offered to future professionals involved in early care.

Key words: knowledge, child development, nutrition, university students. 


\section{INTRODUCCIÓN}

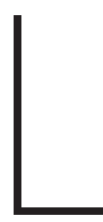

a efectividad del derecho a la educación ha otorgado al título universitario el valor de uno de los logros más importantes en la vida de los individuos. La intención de la sociedad al otorgar la oportunidad de obtener un grado de licenciatura se basa en la posibilidad de ofrecer un mejor servicio a la colectividad? De esta forma las universidades tienen un gran desafío en cuanto a lograr que exista una respuesta a las necesidades reales de la sociedad, oportunidades del mercado laboral y condiciones de salud de la población.

Debido a que los reportes de 2012 en México muestran que el $8.37 \%$ de los niños nacieron con bajo peso, a 9.19\% de los niños nacidos vivos no se les realizó el tamiz neonatal y 29\% de los niños tienen o están en riesgo de tener una discapacidad², por ello es importante que los futuros profesionales que van a atender al binomio madre-niño cuenten con el conocimiento suficiente para resolver los problemas de Desarrollo infantil, Nutrición y Alimentación.

El Desarrollo Infantil es un proceso activo e individualizado para cada niño, caracterizado por la continuidad y los cambios en la adquisición de habilidades motoras, cognitivas, psicosociales y del lenguaje. ${ }^{3}$ Debido a lo cual el periodo prenatal y los primeros cinco años de la infancia son decisivos en el proceso de desarrollo, ahí es donde confluye la interacción de las características biopsicológicas, heredadas genéticamente, con las experiencias que ofrece el entorno. ${ }^{3.4}$

Así mismo el desarrollo cognitivo guarda relación directa con el grado de estimulación ambiental recibido. Una adecuada estimulación sensorial, motriz, cognitiva y afectiva incrementa las conexiones nerviosas y optimiza la función cerebral. Por el contrario, una estimulación insuficiente, incluso en niños normales, contribuye a un desarrollo anatómico y funcional anómalo. ${ }^{5}$
Por otro lado, debido al riesgo de alteraciones relacionadas con la nutrición, es necesario vigilar la ingesta de nutrientes como hierro, ácido fólico, vitaminas del complejo B y calcio durante el embarazo. El ácido fólico y las vitaminas del complejo B son vitales para el desarrollo neurológico fetal y su deficiencia se asocia a espina bífida y defectos del tubo neural; en cuanto al calcio, es indispensable para la formación del sistema óseo y el aporte adecuado de hierro evita anemias. ${ }^{6}$

Además de los macronutrientes, la leche materna tiene factores funcionales que intervienen en la síntesis, la proliferación y la diferenciación celular, en la maduración de órganos, así como sustancias inmunomoduladoras y enzimas digestivas. Por lo que la lactancia materna proporciona una mejor regulación metabólica, una mayor protección inmunitaria, con disminución de la morbilidad infecciosa y una disminución del riesgo de sensibilidad alérgica. Aunque los niños alimentados con leche materna ganan peso más lentamente que los alimentados con fórmulas, datos epidemiológicos asocian la lactancia materna a una menor incidencia de obesidad en la vida adulta?

Diferentes estudios han evaluado los conoci-mientos en la población universitaria, como el de Montero et al., ${ }^{8}$ quienes evaluaron el nivel de conocimientos sobre nutrición en estudiantes de la Universidad de San Pablo en Madrid ${ }^{8}$, Da Silva et al., ${ }^{9}$ abordaron el tema de evaluación del conocimiento sobre Vigilancia de desarrollo infantil en enfermeros, de Brasil?, en Cuba, Díaz et al.,10 evaluaron el conocimiento sobre la atención que otorgan como cuidadores los estudiantes de enfermería ${ }^{10}$, y en Chile, Sotomayor ${ }^{17}$ reportó un estudio sobre percepción de la formación en aspectos de desarrollo en estudiantes de Pedagogía.

Este estudio tiene como objetivo analizar qué saben sobre desarrollo infantil, nutrición y alimentación los estudiantes de las licenciaturas de Nutrición, Enfermería, Medicina, Psicología y Pedagogía. 


\section{MATERIAL Y MÉTODOS}

巨

studio exploratorio mediante la realización de una encuesta semiestructurada en una muestra de población de estudiantes de las licenciaturas de: Enfermería, Nutrición, Medicina, Psicología y Pedagogía de la Universidad Veracruzana campus- Xalapa, Ver.

Participaron 134 estudiantes, 40 hombres y 94 mujeres con una edad media de 20 años \pm 3 meses, siendo la edad mínima de 18 y la máxima de 36 años. Los estudiantes fueron alumnos de segundo y cuarto semestre de nivel licenciatura, 16\% Nutrición, 28\% Enfermería, 22\% Medicina, 16\% Psicología y 17\% Pedagogía.
Se aplicó un cuestionario con preguntas cerradas y semicerradas divididas en 3 apartados: 1) datos generales, 2) 10 preguntas de desarrollo infantil, 1,13 y 3) 18 preguntas de nutrición y alimentación. 14-16

Dadas las características del estudio, la ausencia de intervención, el tratamiento de los datos y la obtención de la muestra no se ha estimado necesario someterlo a un comité ético, siendo requerido el consentimiento informado.

Para procesar los datos obtenidos se utilizó el programa Minitab 15 aplicándose pruebas de Kruskal Wallis.

\section{RESULTADOS}

D e total de estudiantes 30\% consideró que su conocimiento sobre desarrollo infantil es muy poco, $39 \%$ que es bueno y $37 \%$ suficiente.

Según los resultados de la encuesta 95\% ha recibido información acerca de Desarrollo infantil a través de profesionales de la salud, revistas especializadas y medios de comunicación como radio y televisión. Respecto a la búsqueda de información 20\% no la ha buscado, 43\% lo ha realizado a través de internet y 13\% ha consultado a profesionales de la salud.

De total de los estudiantes 50\% reportaron que tienen mucho interés en el tema de Desarrollo infantil y el resto muestran poco o algún interés. 87\% de la población sabe que la forma correcta de evaluar el desarrollo es a través de instrumentos estandarizados, para este caso destaca que las respuestas fueron acertadas para todos los estudiantes de las Licenciaturas en Enfermería y Nutrición.

Del total de los estudiantes $67 \%$ nunca ha evaluado el desarrollo infantil, los alumnos que refirieron mayor frecuencia en evaluaciones fueron los de enfermería (74\%). Es importante resaltar que los alumnos de la licenciatura en Nutrición solo realizan esta actividad a través de la exposición de casos clínicos documentados.

De acuerdo al instrumento, se observó una mediana de 5 de 10 aciertos esperados (tabla 1).

Tabla 1. Distribución de datos de aciertos en el cuestionario de desarrollo infantil por los estudiantes de acuerdo a la Licenciatura.

\begin{tabular}{|c|c|c|c|c|c|c|}
\hline Licenciatura & Promedio & Desv. estándar & Mínimo & Máximo & Moda & Mediana \\
\hline Nutrición & 5 & 1.20 & 3 & 7 & 5 & 5 \\
\hline Enfermería & 4.5 & 1.85 & 0 & 8 & 5 & 5 \\
\hline Medicina & 4.2 & 1.94 & 0 & 8 & 5 & 4.5 \\
\hline Psicología & 4.3 & 1.78 & 1 & 8 & 4.5 & 4 \\
\hline Pedagogía & 4.5 & 1.43 & 2 & 7 & 5 & 5 \\
\hline
\end{tabular}

Fuente: cuestionario sobre Desarrollo Infantil, Nutrición y alimentación.




Las preguntas que se detectaron con menor dificultad fueron las relacionadas a los hitos del desarrollo psicomotor, específicamente los que se referían a las conductas: "el niño camina agarrándose de los muebles o de la mano y se agacha por objetos utilizando una pinza superior", así como la conducta de "gateo".

En el área de lenguaje, los alumnos identificaron en mayor proporción la edad a la que el niño dice las palabras de mamá y papá para referirse a personas específicas. Se observó en la mayoría de los casos que se obtuvieron respuestas correctas en las conductas que se logran después de los seis meses de edad.

De acuerdo con la prueba de Kruskal-Wallis, no se hallaron diferencias significativas por Licenciatura en los conocimientos sobre el desarrollo infantil.

Respecto a los conocimientos sobre nutrición y alimentación todos los estudiantes tuvieron conocimiento insuficiente, con promedio de $2.3 \pm 1.3$ aciertos de 18 (Figura 1).

Figura 1. Distribución de las medianas de aciertos sobre Nutrición y Alimentación de los estudiantes de acuerdo a la licenciatura que cursan.

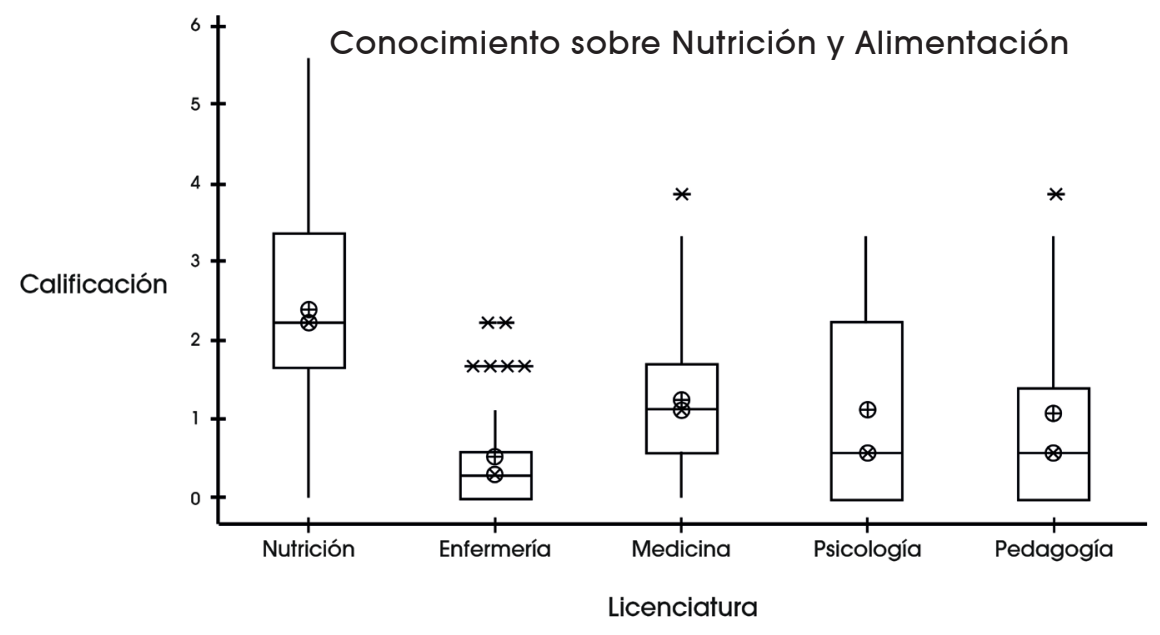

Fuente: Cuestionario sobre Desarrollo Infantil, Nutrición y alimentación.

Se encontró, según la prueba de Kruskal Wallis, que hay diferencias estadísticamente significativas entre los alumnos de las diferentes Licenciaturas al evaluar los conocimientos sobre nutrición durante el embarazo, y alimentación durante el primer año de vida, sin embargo, todos los estudiantes tuvieron un conocimiento insuficiente (tabla 2).

Tabla 2. Distribución de datos de aciertos del cuestionario de Nutrición y alimentación de los estudiantes de acuerdo a la licenciatura.acuerdo a la licenciatura que cursan.

\begin{tabular}{|c|c|c|c|c|}
\hline Licenciatura & Promedio & Mediana & del promedio & $\begin{array}{c}\text { Clasificación } \\
\text { Z }\end{array}$ \\
\hline Nutrición & 21 & 2.2222 & 102.6 & 4.51 \\
\hline Enfermería & 38 & 0.2778 & 44.2 & -4.38 \\
\hline Medicina & 30 & 1.1111 & 74.3 & 1.09 \\
\hline Psicología & 22 & 0.8333 & 69.8 & 0.30 \\
\hline \multirow[t]{2}{*}{ Pedagogía } & 23 & 0.5556 & 62.9 & -0.62 \\
\hline & Total & 134 & & 67.5 \\
\hline
\end{tabular}

Fuente: cuestionario sobre Desarrollo Infantil, Nutrición y alimentación. Enf Neurol Vol. 16. No. 2 mayo - agosto 2017 Nhthr 


\section{DISCUSIÓN}

os procesos complejos de crecimiento y desarrollo inician en la gestación, en el primer año es determinante el aporte adecuado de nutrientes, que tendrá influencia en la composición corporal y definirá los futuros riesgos a la salud $y$, en el segundo año, se especializan las funciones del sistema nervioso, incluyendo las de los órganos sensoriales, que se expresa a través de la maduración y se constata mediante la evaluación del logro de conductas. Para que el desarrollo sea óptimo además de una estructura genética adecuada y la consecución de exigencias biológicas, se requiere la satisfacción de necesidades psicoafectivas y un contexto de estimulación adecuado.

Debido a las evidencias de la participación de los factores genéticos y ambientales en estos procesos se hace necesario conocer, de manera temprana, sí el desarrollo y crecimiento del niño ha sido óptimo o si existieron factores de riesgo. ${ }^{17}$

En la evaluación del crecimiento y desarrollo infantil cobra importancia la profesionalización, la interdisciplinariedad y la transdisciplinariedad convirtiéndose en un desafío para las instituciones formadoras de recursos humanos dirigidas a la atencióntemprana, razón por lo que es indispensable conocer qué saben los estudiantes con respecto a Desarrollo infantil, la Nutrición y la Alimentación.

En este trabajo se logró conocer lo que saben los estudiantes de las licenciaturas en Nutrición, Enfermería, Medicina, Psicología y Pedagogía acerca del Desarrollo infantil Nutrición y alimentación. El resultado coincide con el estudio de Díaz ${ }^{12}$ quien mostró que existía dificultad en los estudiantes universitarios al expresar sus conocimientos. En nuestro caso se observó que el nivel de conocimientos no fue homogéneo entre grupos, aunque las puntuaciones de conocimiento de desarrollo fueron más altas en los estudiantes de Enfermería y Medicina con relación a los demás grupos, en todos los estudiantes el conocimiento acerca de los hitos del desarrollo fue inferior a lo recomendado.

Cabe destacar que la mayoría de los estudiantes refirió tener conocimiento, sin embargo, esta información fue sobreestimada al constatarse en la evaluación de sus respuestas. En los planes de estudio de las disciplinas a la que pertenecen los estudiantes se abordan temas de desarrollo infantil, sin embargo, se desconoce cuáles son las causas por las que los estudiantes no logran demostrar dichos conocimientos.

Aunque los resultados obtenidos en la evaluación son similares a lo observado en otros estudios, se identificó que los estudiantes alcanzaron calificaciones menores al 50\% de los aciertos y tuvieron problemas para identificar con precisión los hitos del desarrollo.

Las respuestas sobre las recomendaciones de alimentación y Nutrición fueron más altas en estudiantes de Nutrición sobre todo en aspectos de Ablactación y de las ingestas diarias recomendadas (IDR) de ácido fólico durante el embarazo, sin embargo, los conocimientos de éste rubro son insuficientes en todos los grupos.

Al igual que el estudio de Da Silva ${ }^{17}$ quien encuestó a Enfermeros sobre el conocimiento de Desarrollo Infantil, en nuestro estudio se observó que aunque los estudiantes tuvieron conocimiento insuficiente, manifestaron interés sobre el tema y la forma de aplicarlo a su práctica laboral.

Por lo anterior, se debe promover que los estudiantes se apropien del conocimiento ${ }^{18}$ favoreciendo que evalúen aspectos de Desarrollo Infantil, Alimentación y Nutrición de manera cotidiana, considerando

\section{6}


la interacción con las madres de familia a fin de que experimenten afinidad por el tema, decidan profundizarlo y de esta manera mejoren su desempeño profesional desarrollando las competencias que les permitan identificar a los niños que presentan trastornos en su desarrollo o tienen riesgo de padecerlos.

Se sugiere la creación de una unidad multidisciplinaria de Nutrición y Desarrollo infantil, como estrategia educativa, donde los alumnos de las disciplinas evaluadas asistan a un espacio práctico, en el que se les de la oportunidad de observar y evaluar los procesos de desarrollo y nutrición en un contexto interdisciplinario, a fin de perfilar su desempeño profesional en la atención temprana.

\section{REFERENCIAS BIBLIOGRÁFICAS}

1. Carpizo, J. La Constitución Mexicana de 1917. Porrúa, 2000

2. De Castro, F., Allen-Leigh, B., Katz, G., Salvador-Carulla, L., \& Lazcano-Ponce, E. Indicadores de bienestar y desarrollo infantil en México. Salud Pública de México, 2013; 55, S267-\$275.

3. Souza, J. M. D., \& Veríssimo, M. D. L. Ó. R. Child development: analysis of a new concept. Revista latino-americana de enfermagem. 2015; 23(6), 1097-1104.

4. Lipina, S. J., \& Segretin, M. S. 6000 días más: evidencia neurocientífica acerca del impacto de la pobreza infantil. Psicología Educativa. 2015; 21 (2), 107-116.

5. Quino, A. C., \& Barreto, P. Motor development in children with malnutrition in Tunja, Boyacá. Revista Facultad Nacional de Salud Pública. 2015; 33(1), 15-21.

6. Barón, M, Peña, E, Sánchez, A, \& Solano, L. Estado de ácido fólico en embarazadas adolescentes y adultas en el primer trimestre del embarazo. Anales Venezolanos de Nutrición, (Internet). 2002 (consulta el 10 de diciembre de 2016); 15(2), 87-93. Disponible en: http://www.scielo.org.ve/ scielo.php?script=sci_arttext\&pid=S0798-07522002000200004\&lng=es\&tIng=es.

7. Gámez Rodríguez M.E. Nutrición y salud. Coordinadores: Briones R. Rosendo, Vázquez Montiel S y Vélez Pliego M. Benemérita Universidad Autónoma de Puebla. Dirección de Fomento Editorial; 2008.

8. Montero B. A., Úbeda M N., \& García G A. Evaluación de los hábitos alimentarios de una población de estudiantes universitarios en relación con sus conocimientos nutricionales. Nutrición Hospitalaria. 2006; 27 (4), 466-473.

9. da Silva A. P., Collet, N., Eickmann, S. H., \& de Carvalho L, M. Vigilância do desenvolvimento infantil: estudo de intervenção com enfermeiros da Estratégia Saúde da Família. Revista Latino-Americana de Enfermagem 2015; 23(5), 954-962

10. Díaz Aguilar, R., Amador Morán, R., Alonso Uría, R. M., Campo González. A., Mederos Dueñas, M., \& Oria Saavedra, M. (2015). Evaluación del conocimiento del Proceso de Atención de Enfermería: Estudiantes de licenciatura de Enfermería. Facultad "Lidia Doce". Enfermería Universitaria. 2013: 12(1), 36-40.
11. Sotomayor-E, C. Coloma T, C. J. Parodi S, G, Ibáñez O, R, Cavada H. P. \& Gysling C., J. Percepción de los estudiantes de pedagogía sobre su formación inicial. magis, Revista Internacional de Investigación en Educación. 2013; 5 (11), 375-392

12. Bayley, N. Bayley Scales of Infant Development. The Psychological Corporation. USA. 1993

13. Amado AM, Velasco CA. Hincapié $O$, Galindo A. Valoración Motora Social, del Habla y Comprensión del Lenguaje del Lactante Menor Desnutrido por medio de la Prueba de Munich. Revista Gastrohnup. 2003, Volumen 5 Número 1 P. 19-2

14. Combs, GThe Vitamins: fundamental aspects in nutrition and health Elsevier Academic Press, Burlington. 2008; Third Edition. Pp 359. consultation. Bangkok, Thailand

15. Organización Panamericana de la Salud. La alimentación del lactante y del niño pequeño: Capítulo Modelo para libros de texto dirigidos a estudiantes de medicina y otras ciencias de la salud. 2010; ISBN: 978-92-7533094-4 NLM WS120 Washington, D.C.

16. Rodríguez, G. Martín D. Nutrición y Neurología. 2015; Akadia editorial. Argentina.

17. Moreno, T. El papel del conocimiento escolar universitario. Acta Médica Colombiana. 2015; 40(3), 241.

18. SSA Norma Oficial Mexicana NOM-007-SSA2-2016, para la atención de la mujer durante el embarazo, parto y puerperio, y de la persona recién nacida. 2016 